\title{
Transient ventilation dynamics following a change in strength of a point source of heat
}

\author{
D. J. BOWER ${ }^{1} \uparrow$, C. P. CAULFIELD ${ }^{1,2}$, S. D. FITZGERALD ${ }^{1}$ \\ AND A. W. WOODS \\ ${ }^{1}$ BP Institute, University of Cambridge, Madingley Road, Cambridge, CB3 0EZ, UK \\ ${ }^{2}$ Department of Applied Mathematics and Theoretical Physics, University of Cambridge, Centre for \\ Mathematical Sciences, Wilberforce Road, Cambridge, CB3 0WA, UK \\ dan@caltech.edu, c.p.caulfield@bpi.cam.ac.uk, shaun@bpi.cam.ac.uk, andy@bpi.cam.ac.uk
}

(Received 11 May 2007, and in revised form 26 June 2008)

We investigate the transient ventilation flow within a confined ventilated space, with high- and low-level openings, when the strength of a low-level point source of heat is changed instantaneously. The steady-flow regime in the space involves a turbulent buoyant plume, which rises from the point source to a well-mixed warm upper layer. The steady-state height of the interface between this layer and the lower layer of exterior fluid is independent of the heat flux, but the upper layer becomes progressively warmer with heat flux. New analogue laboratory experiments of the transient adjustment between steady states identify that if the heat flux is increased, the continuing plume propagates to the top of the room forming a new, warmer layer. This layer gradually deepens, and as the turbulent plume entrains fluid from the original warm layer, the original layer is gradually depleted and disappears, and a new steady state is established. In contrast, if the source buoyancy flux is decreased, the continuing plume is cooler than the original plume, so that on reaching the interface it is of intermediate density between the original warm layer and the external fluid. The plume supplies a new intermediate layer, which gradually deepens with the continuing flow. In turn, the original upper layer becomes depleted, both as a result of being vented through the upper opening of the space, but also due to some penetrative entrainment of this layer by the plume, as the plume overshoots the interface before falling back to supply the new intermediate layer. We develop quantitative models which are in good accord with our experimental data, by combining classical plume theory with models of the penetrative entrainment for the case of a decrease in heating. Typically, we find that the effect of penetrative entrainment on the density of the intruding layer is relatively weak, provided the change in source strength is sufficiently large. However, penetrative entrainment measurably increases the rate at which the depth of the draining layer decreases. We conclude with a discussion of the importance of these results for the control of naturally ventilated spaces.

\section{Introduction}

When there is a localized source of heating at the base of an enclosed space, with high- and low-level openings to a uniform exterior, a two-layer natural ventilation flow, known as the 'emptying filling box' develops. In steady state, a turbulent buoyant

$\dagger$ Present address: Seismological Laboratory, California Institute of Technology, Pasadena, CA 91125, USA. 


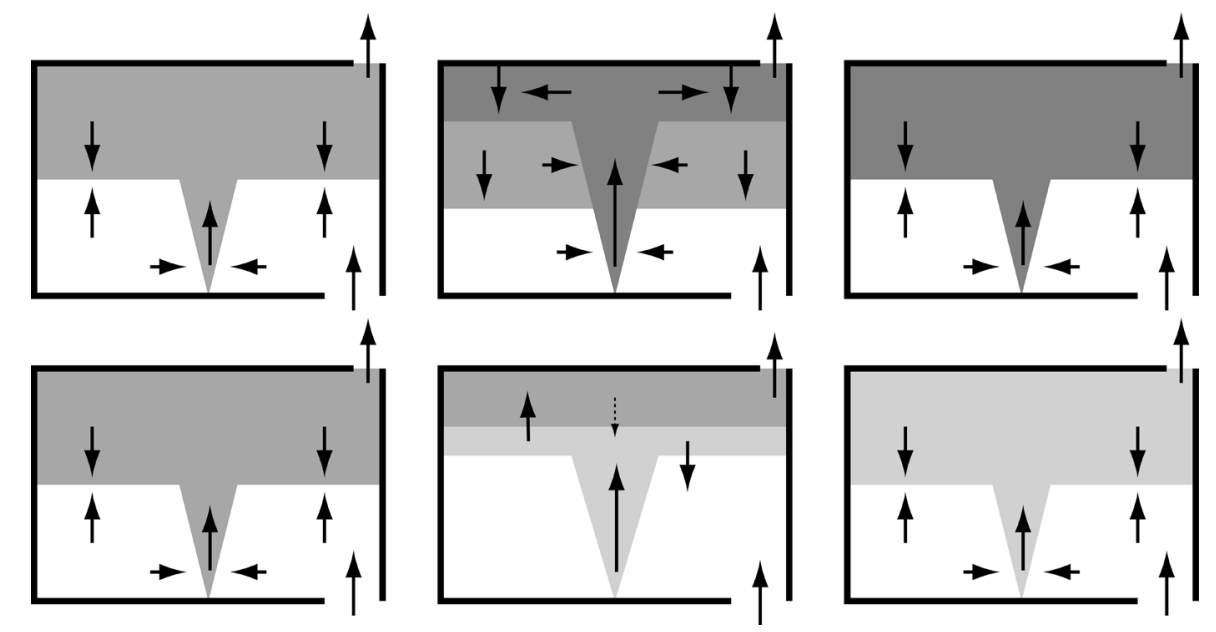

FIGURE 1. Schematic representation of the transient flow when the source buoyancy is either discontinuously: increased (upper panels, and so the new layer fills the room from above); or decreased (lower panels, and so the new layer intrudes from below). The original buoyant layer is shaded in light grey in each case, and flow directions are shown with arrows. In the intruding case, the possibility for penetrative entrainment is represented by the small dashed downward arrow between the original and the intruding layer above the plume.

plume rises from the heat source to an upper well-mixed layer. This plume entrains fluid of ambient density from the lower layer. The depth of the interface corresponds to the level at which the volume flux in the plume equals the ventilation volume flux. With a pure source of heating, this depth is independent of the heat source, and only depends on the areas of the ventilation openings, the depth of the room and the coefficient of entrainment associated with the plume (Linden, Lane-Serff \& Smeed 1990). However, the temperature of the warm upper layer does increase with the strength of the heat source (Linden et al. 1990). In this paper we examine the process by which the flow evolves from one such steady state to another as a result of a change in the heating load.

Two qualitatively different transient flows can occur, depending on whether the source heat flux increases or decreases, as shown schematically in figure 1. If the source heat flux increases, the temperature and intensity of the plume increases. The plume is buoyant relative to the original upper warm layer and hence rises to the top of the space. Here it forms a new hot layer, which gradually deepens with time, while the original warm layer is entrained by this plume thereby becoming depleted, as shown in the upper panels of figure 1. Eventually, the original warm layer disappears, and the new hot layer attains the depth of the original upper layer. We refer to this flow regime as the 'filling' regime. We note that Kaye \& Hunt (2004) have described the limiting case of the transition from an initially unheated room to a heated room, and we compare our results with that case for reference.

Conversely, if the source heat flux is decreased, the plume becomes cooler and less intense, so that on reaching the original upper warm layer, the continuing plume is now cold and dense. The plume then intrudes at this level to form a new layer, as shown in the lower panels of figure 1. The original warm upper layer may then become depleted owing to two processes. Firstly, it drains through the top opening as a continuation of the upward ventilation flow. Secondly, the continuing turbulent plume may entrain some of the upper-layer fluid since, although it is relatively dense, 
it can overshoot the interface, thereby mixing with some of the original upper-layer fluid, before falling back into the lower layer (Baines 1975; Baines, Turner \& Campbell 1990; Bloomfield \& Kerr 2000; Linden 1973; Kumagai 1984; Lin \& Linden 2005; McDougall 1981). We refer to this regime as the 'intruding' regime.

In $\S 2$, we develop idealized models to describe both the dynamics of the 'filling' (increased heating) and 'intruding' (decreased heating) flows. For simplicity, we assume that the change in the heat load is instantaneous, and also that the buoyant layers that form are well mixed, which is typically a good approximation (see Caulfield \& Woods 2002; Flynn \& Caulfield 2006 for a fuller discussion of the well-mixed approximation, and Bolster \& Caulfield 2008 for a discussion of the effects of continuous variation in heat load). In $\S 3$, we present some new analogue experiments which illustrate and quantify the transient flow in both cases of increasing and decreasing source buoyancy fluxes.

We compare the predictions of our various models with the results of the experiments to test our modelling approach, in particular the assumption that the layers are well mixed, and also to test whether the observed penetrative entrainment plays a significant dynamical role in intruding flows. For intruding flows, we find that including penetrative entrainment improves the prediction of the observed evolution of the interface between the original and intruding layers. However, due in part to the fact that the layers are not completely well mixed, the typical density in the intruding layer is actually usually better modelled using the assumption that no entrainment takes place. The two models with and without entrainment can usefully be thought of as end-members between which the real flow evolution occurs. Finally, in $\S 4$, we draw some conclusions.

\section{Model development}

In modelling the flow, we combine the theory of turbulent buoyant plumes (Morton Taylor \& Turner 1956) in the lower layer, with a model of the buoyant ascent of fluid through the space and through the ventilation openings (Linden et al. 1990), allowing for the transient evolution of the system through the inclusion of either a new upper layer for the filling regime or a new intermediate layer for the intruding regime. In the intruding regime, we also allow for the possibility of penetrative entrainment associated with the plume rising into the original warm upper layer. We are particularly interested in investigating whether the penetrative entrainment is significant dynamically, and so we also allow for the calculation of flow evolution with no penetrative entrainment. We assume that each of the layers is thoroughly well mixed, and that the interfaces between the layers are sharp. These are always going to be approximations to reality, particularly when considering the effects of penetrative entrainment, an issue we return to in $\S 3$ when considering our experimental results.

We note that in the filling regime, the continuing plume rises through both the lower layer of ambient fluid and the original warm layer. Since these have different densities, the plume experiences a discrete change in buoyancy relative to the surrounding fluid as it passes across the interface. In this situation, it is most accurate to solve the conservation equations for the turbulent buoyant plume above the interface in order to calculate the mass flux supplied to the new upper layer.

\subsection{Plume model}

Equations for the conservation of volume flux $Q$ and specific momentum flux $M$ of a turbulent buoyant plume with buoyancy flux $F$ in a uniform environment were 
developed by Morton et al. (1956) and take the form

$$
\frac{\mathrm{d}}{\mathrm{d} z} Q=2 \alpha \pi^{1 / 2} M^{1 / 2} ; \frac{\mathrm{d}}{\mathrm{d} z} M=\frac{F Q}{M} ; \frac{\mathrm{d}}{\mathrm{d} z} F=0 ;
$$

where $\alpha \sim 0.1$ is an empirically determined entrainment constant. For a ventilation flow in a confined space of height $H$ it is convenient to scale these equations using the similarity solutions derived by Morton et al. (1956) for a turbulent buoyant plume rising from a source of pure buoyancy $F_{o}$ through a uniform ambient of height $H$. In these solutions, the volume flux $Q_{o}(H)$ and the specific momentum flux $M_{o}(H)$ of the original plume with source buoyancy flux $F_{o}$ at height $H$ are given by

$$
Q_{o}(H)=\frac{6 \alpha}{5}\left(\frac{9 \alpha \pi^{2} F_{o} H^{5}}{10}\right)^{1 / 3}=\lambda\left(F_{o} H^{5}\right)^{1 / 3} ; M_{o}(H)=\left(\frac{9 \alpha \pi^{1 / 2} F_{o} H^{2}}{10}\right)^{2 / 3} .
$$

Also, we scale the height $z$ by the depth of the space $H$, defining $\zeta=z / H$. The dimensionless buoyancy flux, mass flux and momentum flux then take the form

$$
f=\frac{F}{F_{o}}=\hat{g} q ; q=\frac{Q}{Q_{o}(H)}=\frac{Q}{\lambda F_{o}^{1 / 3} H^{5 / 3}} ; m=\frac{M}{(9 \alpha / 10)^{2 / 3} \pi^{1 / 3} F_{o}^{2 / 3} H^{4 / 3}}, \hat{g}=\frac{\lambda g^{\prime} H^{5 / 3}}{F_{o}^{2 / 3}},
$$

where $g^{\prime}$ is the reduced gravity $g^{\prime}=g\left(\rho_{a}-\rho\right) / \rho_{a}$ relative to ambient fluid of density $\rho_{a}$. Finally, it is convenient to scale time with the 'filling box' time, i.e. the time required to fill the enclosure by a plume with volume flux $Q_{o}(H)$. The filling box time $T_{o}$ is defined as

$$
T_{o}=\frac{A_{c} H}{Q_{o}(H)},
$$

with $A_{c}$ representing the cross-sectional area of the room, so that the dimensionless time $\tau=t / T_{o}$.

The dimensionless equations for the plume in an ambient of constant density in an arbitrary region $\zeta_{1}<\zeta<\zeta_{2}$ then take the form

$$
\frac{\mathrm{d}}{\mathrm{d} \zeta} q=\frac{5}{3} m^{1 / 2} ; \frac{\mathrm{d}}{\mathrm{d} \zeta} m=\frac{4}{3} \frac{f q}{m} \rightarrow \frac{\mathrm{d}}{\mathrm{d} \zeta} q=\frac{5}{3}\left[f\left(q^{2}-q_{c}^{2}\right)+m_{c}^{5 / 2}\right]^{1 / 5},
$$

where $m\left(\zeta_{1}\right)=m_{c}$ and $q\left(\zeta_{1}\right)=q_{c}$ and, in the region $\zeta_{1}<\zeta<\zeta_{2}$ the plume buoyancy flux is $f$.

\subsection{Ventilation flow}

\subsubsection{Filling regime}

In the case of an instantaneous increase in heat load (as shown in figure $2 a$ ), the dimensionless ventilation flow, $q_{t}=Q_{t} / Q_{o}(H)$, through the high-level or 'top' opening (see figure $2 a$ ) depends on the depth of the new buoyant layer with dimensionless reduced gravity $\hat{g}_{f}>\hat{g}_{o}$, located in the region $1>\zeta>\zeta_{f}(\tau)$, and the original buoyant layer, of dimensionless reduced gravity $\hat{g}_{o}$, located in the region $\zeta_{o}(\tau)<\zeta<\zeta_{f}(\tau)$, according to the relation (Linden et al. 1990)

$$
\frac{Q_{t}}{Q_{o}(H)}=q_{t}=\frac{A_{\star}}{\lambda^{3 / 2} H^{2}}\left[\hat{g}_{o}\left(\zeta_{f}-\zeta_{o}\right)+\hat{g}_{f}\left(1-\zeta_{f}\right)\right]^{1 / 2} \text {. }
$$

In this expression $A_{\star}$ is the effective opening area

$$
A_{\star}^{2}=\frac{2 A_{t}^{2} A_{b}^{2}}{A_{t}^{2}+A_{b}^{2}},
$$



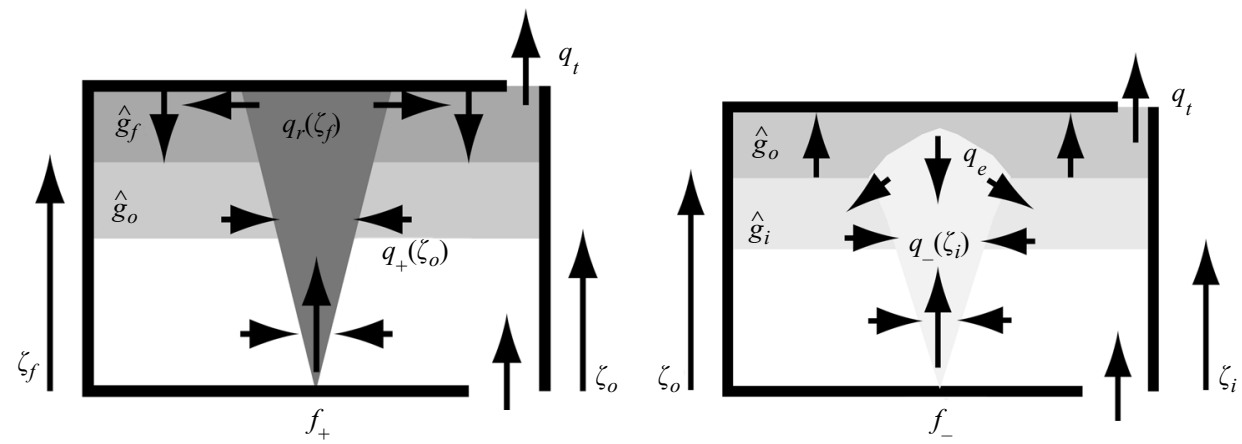

FIgURE 2. Schematics of the $(a)$ 'filling' regime and $(b)$ 'intruding regime' illustrating the various dimensionless variables used.

where $A_{t}$ and $A_{b}$ are the product of the areas of the top and bottom openings and their discharge coefficients (Linden et al. 1990).

\subsubsection{Intruding regime}

In the case of a reduction in the heat load (see figure $2 b$ ), the new layer, with reduced gravity $\hat{g}_{i}<\hat{g}_{o}$ ) intrudes below the original layer in the region $\zeta_{i}(\tau)<\zeta<\zeta_{o}(\tau)$. Now, the original warm layer, with reduced gravity $\hat{g}_{o}$ lies in the region $\zeta_{o}<\zeta<1$. In this regime, the ventilation flux is given by

$$
q_{t}=\frac{A_{\star}}{\lambda^{3 / 2} H^{2}}\left[\hat{g}_{o}\left(1-\zeta_{o}\right)+\hat{g}_{i}\left(\zeta_{o}-\zeta_{i}\right)\right]^{1 / 2} .
$$

\subsection{Steady state}

At final steady state, $\zeta_{o}=\zeta_{f}=\zeta_{\infty}$ for a filling flow associated with an increase in the heating. Conversely, $\zeta_{o}=1, \zeta_{i}=\zeta_{\infty}$ for an intruding flow associated with a decreased buoyancy flux. In both cases, if we match the volume flux in the plume, as given by the original similarity solutions of Morton et al. (1956), at the interface, $\zeta=\zeta_{\infty}$ with the ventilation flux $q_{t}$, we find

$$
\frac{\zeta_{\infty}^{5}}{1-\zeta_{\infty}}=\frac{A_{\star}^{2}}{\lambda^{3} H^{4}}
$$

This illustrates that the final interface location coincides with the original interface location, and is purely a function of the opening areas, room height and entrainment coefficient as originally derived by Linden et al. (1990).

\subsection{Transient dynamics of the filling flow}

We assume that for $\tau=t / T_{o}<0$, the original source is a source of buoyancy flux $f=1$ alone, which, for simplicity, can be modelled using the point source similarity solution given in (2.2). Therefore, initially $q_{o}(\zeta)=\zeta^{5 / 3}, m_{o}(\zeta)=\zeta^{4 / 3}$. At $\tau=0^{+}$, the source buoyancy flux is increased to $f_{+}>1$. The plume fluid is buoyant when it arrives at the original upper layer, and so it propagates to the ceiling, where it spreads out and forms a deepening layer which fills the room from above (figure $2 a$ ).

Assuming that the filling layer is well mixed at all times, the evolution equations are

$$
\frac{\mathrm{d}}{\mathrm{d} \tau} \zeta_{o}=-q_{+}\left(\zeta_{o}\right)+q_{t}, \quad \frac{\mathrm{d}}{\mathrm{d} \tau} \zeta_{f}=-q_{r}\left(\zeta_{f}\right)+q_{t}, \quad \frac{\mathrm{d}}{\mathrm{d} \tau} \hat{g}_{f}=\frac{\left[\hat{g}_{r}\left(\zeta_{f}\right)-\hat{g}_{f}\right] q_{r}\left(\zeta_{f}\right)}{\left(1-\zeta_{f}\right)}
$$


Assuming that the new upper layer remains well mixed, we obtain the equation for $\hat{g}_{f}$ by considering the evolution of the total buoyancy in the upper layer $\left(1-\zeta_{f}\right) \hat{g}_{f}$, and then using the equation for $\zeta_{f}$ to eliminate $q_{t}$. The volume flux $q_{r}$ of the plume at $\zeta=\zeta_{f}$ is determined in two stages. For $0<\zeta<\zeta_{o}$, we use the classical predictions for the volume flux and momentum flux of a plume rising in a uniform environment, $q_{+}\left(\zeta_{o}\right)=f_{+}^{1 / 3} \zeta_{o}^{5 / 3}$ and $m_{+}\left(\zeta_{o}\right)=f_{+}^{2 / 3} \zeta_{o}^{4 / 3}$. Once the buoyant plume enters the original buoyant layer, the driving buoyancy flux of the plume is reduced from

$$
f=f_{+}=\left[\rho_{a}-\rho_{+}\left(\zeta_{o}\right)\right] /\left[\rho_{a}-\rho_{o}(H)\right] q_{+}\left(\zeta_{o}\right),
$$

at $\zeta=\zeta_{o-}$, to

$$
f=f_{r}=\frac{\left[\rho_{o}-\rho_{+}\left(\zeta_{o}\right)\right]}{\left[\rho_{a}-\rho_{o}(H)\right]} q_{+}\left(\zeta_{o}\right)=f_{+}-\hat{g}_{o} q_{+}\left(\zeta_{o}\right)>0,
$$

at $\zeta=\zeta_{o+}$. The volume flux $q_{r}\left(\zeta_{f}\right)$ for the plume with reduced buoyancy flux $f=f_{r}$ is then determined by solving (2.5) in the region $\zeta_{0}<\zeta<\zeta_{f}$ with the boundary conditions $q_{c}=q_{+}\left(\zeta_{o}\right), m_{c}=m_{+}\left(\zeta_{o}\right)$. This two-stage approach is essential since the reduction in the buoyancy flux at the original interface, $\zeta_{o}$, causes the plume volume and momentum flux to diverge from the classic similarity solution (see Caulfield \& Woods 1995; Hunt \& Kaye 2001; Morton 1959). Using (2.12), the reduced gravity of the plume fluid relative to the ambient at the filling layer interface $\hat{g}_{r}\left(\zeta_{f}\right)$ is given by

$$
\hat{g}_{r}\left(\zeta_{f}\right)=\frac{f_{r}}{q_{r}\left(\zeta_{f}\right)}+\hat{g}_{o}
$$

and so all the quantities on the right-hand sides of (2.10) can be determined. In solving these equations, the appropriate initial conditions for the interface locations are $\zeta_{o}(0)=\zeta_{\infty}, \zeta_{f}(0)=1$. The initial reduced gravity of the filling layer $\hat{g}_{f}(0)=\hat{g}_{r}(1)$, where $\hat{g}_{r}(1)$ is determined by using (2.13) in (2.5) which are then integrated all the way from the initial location of the original interface, $\zeta=\zeta_{\infty}$, to the ceiling, $\zeta=1$.

The typical transient behaviour is shown in figure $3(a)$. For clarity, we have chosen $\zeta_{\infty}=1 / 2$, and so the steady-state interface is always at the midpoint of the room. We also choose $f_{+}=5$ representing a five-fold increase in the heating rate. On the figure, the evolution of the location of original interface $\zeta_{o}$ is plotted with a thick line, and the filling interface $\zeta_{f}$ with a thin line, shading (with an intermediate shade of grey) the shrinking region occupied by original fluid. The filling region of the new more buoyant fluid is shaded in darker grey. For comparison, the dotted line illustrates the evolution of the depth of the filling interface $\zeta_{f}$ in the different situation in which the room is initially all filled with ambient fluid. This corresponds to the model described by Kaye \& Hunt (2004).

The depth of the lower surface of the original buoyant layer initially descends. This is because just after turning up the heating, at the original depth $\zeta_{o}(0)$ of the warm upper layer, the volume flux in the new plume is greater than that in the original plume. However, the ventilation flow is still controlled by the original warm upper layer. Since this ventilation flow equals the flux in the original weaker plume, the flow of the stronger plume causes the original interface $\zeta_{o}$ to descend. Since the draining flow $q_{t}$ through the top opening is enhanced by the presence of the original layer, the depth of the new filling layer increases more slowly than in the case with no original warm layer (the dashed line). Also, although the lower interface of the original warm fluid layer does descend, the lower interface of the new hot layer converges monotonically from above to the steady-state location. However, comparison of the dashed and solid line indicates that with a large increase in the heating, the original 

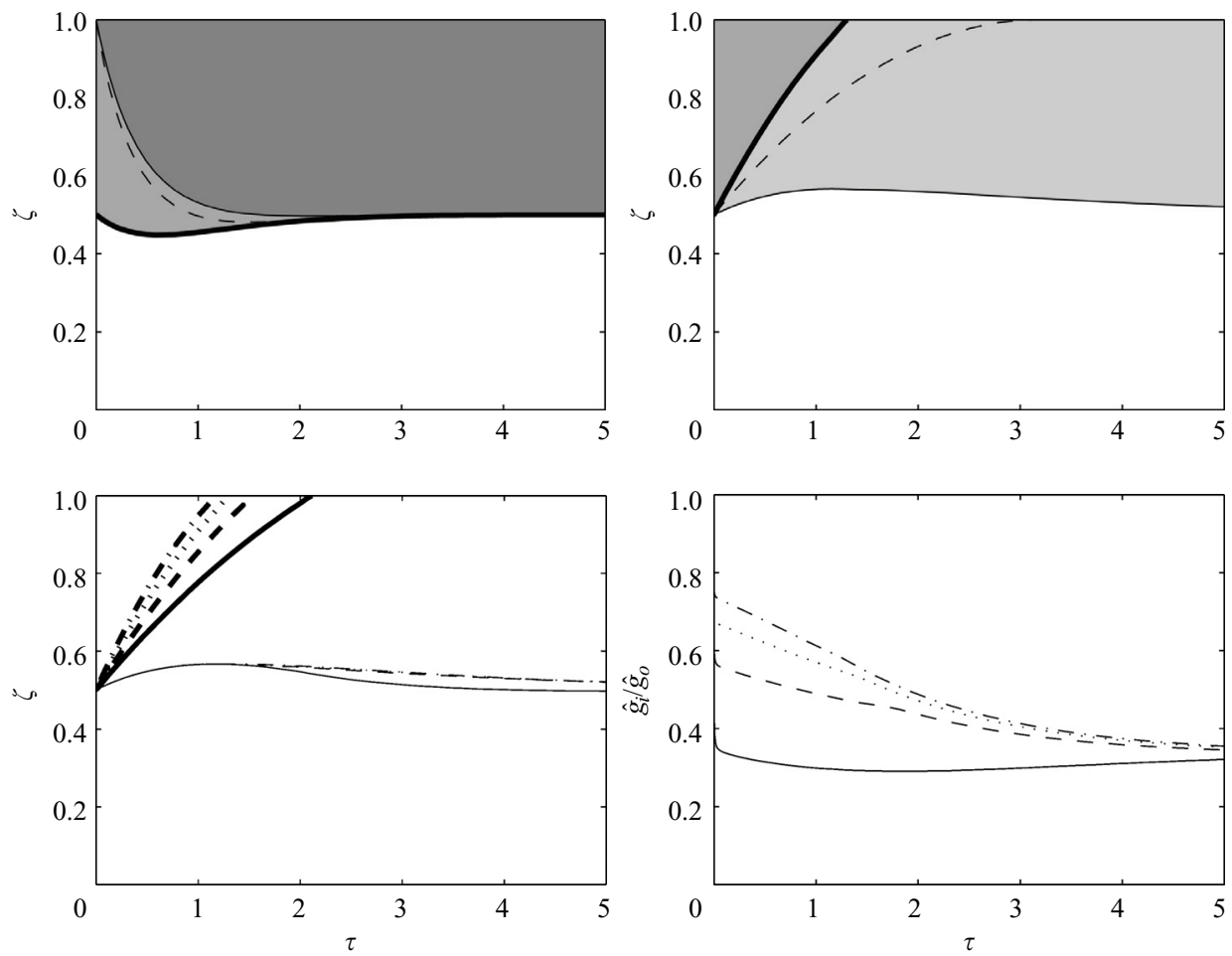

FIGURE 3. Variation with time of various properties of flows with sources of buoyancy alone and $\zeta_{\infty}=1 / 2$. (a) Original interface location $\zeta_{o}$ (plotted with a thick solid line), filling interface location $\zeta_{f}$ (thin solid line) and transient filling box interface location with no original layer (dashed line) for a filling flow with increased source buoyancy flux $f_{+}=5$. The medium shaded region shows the part of the room occupied by the buoyant layer from the original plume, while the darker shaded region shows the more buoyant layer from the plume with increased buoyancy flux $f_{+}>1$. $(b)$ Original interface location $\zeta_{o}$ (plotted with a thick solid line), intruding interface location $\zeta_{i}$ (thin solid line) and a draining original layer in the absence of a plume (dashed line) for an intruding flow with decreased source buoyancy flux $f_{-}=1 / 5$, and entrainment parameter $E=1$. The lightly shaded region is the less buoyant intruding layer from the plume with decreased buoyancy flux $f_{-}<1$, while the medium shaded region shows the part of the room occupied by the buoyant layer from the original plume. (c) The original interface location $\zeta_{o}$ (thick lines) and intruding interface location $\zeta_{i}$ (thin lines) for intruding (decreased buoyancy flux) flows with $f_{-}=1 / 5$, and: $E=0$ (solid lines); $E=0.5$ (dashed lines); $E=1$ (dotted lines); $E=1.5$ (dot-dashed lines). (d) Relative reduced gravity $\hat{g}_{i} / \hat{g}_{o}$ of the intruding layer for intruding (decreased buoyancy flux) flows with $f_{-}=1 / 5$, and: $E=0$ (solid lines); $E=0.5$ (dashed lines); $E=1$ (dotted lines); $E=1.5$ (dot-dashed lines).

warm layer has a secondary influence on the flow. As a result, the transient adjustment to steady state occurs over the filling box time scale associated with the new plume of increased heat flux.

\subsection{Transient dynamics of the intruding flow}

If the heat load is decreased instantaneously to $f_{-}<1$, as illustrated in the lower panels of figure 1, then the original layer will eventually be replaced by a layer of the same depth $1-\zeta_{\infty}$ with a reduced gravity $\hat{g}_{i} \rightarrow f_{-}^{2 / 3} \zeta_{\infty}^{-5 / 3}$ (see figure $2 b$ ). In the transient flow, there are again three layers: a lower layer $0<\zeta<\zeta_{0}$ of ambient fluid (reduced gravity $\hat{g}_{l}=0$ ); the new intruding layer of buoyancy $\hat{g}_{i}$ in the region 
$\zeta_{i}(\tau)<\zeta<\zeta_{o}(\tau)$ and the original layer of warm fluid in the region $\zeta_{o}(\tau)<\zeta<1$ with reduced gravity $\hat{g}_{o}$.

Again, by conservation of mass at the two interfaces, the evolution equations take the form

$$
\frac{\mathrm{d}}{\mathrm{d} \tau} \zeta_{o}=q_{e}+q_{t}, \quad \frac{\mathrm{d}}{\mathrm{d} \tau} \zeta_{i}=-q_{-}\left(\zeta_{i}\right)+q_{t}, \quad \frac{\mathrm{d}}{\mathrm{d} \tau} \hat{g}_{i}=\frac{f_{-}-\hat{g}_{i} q_{-}\left(\zeta_{i}\right)+\left[\hat{g}_{o}-\hat{g}_{i}\right] q_{e}}{\left(\zeta_{o}-\zeta_{i}\right)}
$$

The lower interface of the original layer of buoyant fluid rises as a result of both the draining flow $q_{t}$ through the top opening and the penetrative entrainment flux $q_{e}$. The entrainment flux is caused by the overshoot of the relatively dense plume into the original buoyant layer, since before collapsing back into the intruding layer the plume mixes with some of the upper-layer fluid. The reduced gravity of the intruding layer is affected both by the density of the arriving plume fluid, and also by the density of the fluid entrained from the upper original layer by the plume. The volume flux at the lower interface of the new intruding layer, $q_{-}\left(\zeta_{i}\right)$, is given by the classical similarity solution for an ideal source of buoyancy flux, $f_{-}$using $(2.5)$, i.e.

$$
q_{-}\left(\zeta_{i}\right)=f_{-}^{1 / 3} \zeta_{i}^{5 / 3}=q_{i}
$$

The draining volume flux $q_{t}$ is given by (2.8).

If we assume that there is no penetrative entrainment, $q_{e}=0$ in (2.14). In this case (2.14) reduces to a simple form: the intruding layer is made up of fluid purely from the new plume with reduced buoyancy flux, and the original layer reduces in depth through draining alone. On the other hand, if there actually is penetrative entrainment, it is apparent from (2.14) that penetrative entrainment increases $\hat{g}_{i}$ since $\hat{g}_{o}>\hat{g}_{i}$ (and thus reduces the density of the intruding layer) thus increasing the rate at which the original layer is lost. To complete the mathematical description of the flow in this circumstance, we need a model for $q_{e}$, the volume flux entrained from the original warm layer into the new intruding layer by the overshooting of the plume. As the plume enters the new intermediate layer, at $\zeta=\zeta_{i}$, it is actually a little denser than this layer owing to the earlier penetrative entrainment of the much more buoyant upper layer into this layer. The plume therefore rises into the new intruding layer as a relatively dense fountain. If the momentum of the plume is sufficient, it will continue upwards to the original buoyant layer at $\zeta=\zeta_{o}(\tau)$, above which it may then entrain some of the hot original upper layer.

In the appendix, we develop a simplified model for $q_{e}$, building on much previous work (see Baines 1975; Baines, Turner \& Campbell 1990; Bloomfield \& Kerr 2000; Kumagai 1984; Linden 1973; McDougall 1981). We allow for the fact that the fountain in general rises through two layers in which it is dense: the new intruding layer and the original buoyant layer. Therefore, it is necessary to apportion the entrainment by the fountain between the original buoyant layer (contributing to $q_{e}$ ) and the entrainment in the intruding layer, which just enhances the likelihood that the intruding layer is well mixed. Therefore, as discussed in more detail in the appendix, provided the fountain rise height in the two layers $\zeta_{m}$ is greater than the depth of the intruding layer $\zeta_{o}-\zeta_{i}$, we assume that

$$
q_{e}=E q_{i}\left(1-\frac{\left[\zeta_{o}-\zeta_{i}\right]}{\zeta_{m}}\right)
$$

where the (assumed universal) constant $E$ is empirically determined.

The appropriate initial conditions for a transient intruding flow problem are $\zeta_{o}(0)=\zeta_{i}(0)=\zeta_{\infty}$ and $\hat{g}_{i}(0)=\hat{g}_{-}\left(\zeta_{\infty}\right)=f_{-} / q_{-}\left(\zeta_{\infty}\right)$. A typical transient evolution, 
according to the model (2.14), is shown in figure $3(b)$ for a pure source of heating, and we have again set $\zeta_{\infty}=1 / 2$, and so the steady-state interface is always at the midpoint of the room. In this example, we assume that the heat flux decreases by a factor of five, so $f_{-}=1 / 5$, to complement the earlier case in which the heat flux increases by a factor of five. In this illustrative calculation, we show the model predictions using two different (assumed universal) values of $E$ : the purely intruding case where we model the flow with $E=0$, and a case where we model the effect of non-zero penetrative entrainment by setting $E=1$. These two cases may be considered as end-members, bracketing the experimental behaviour which is actually observed, and discussed in $\S 3$. On the figure, the evolution of the location of the original interface $\zeta_{o}$ with time is also plotted using a thick solid line, and the location of the intruding interface $\zeta_{i}$ with time is plotted using a thin line. The region occupied by original fluid is shaded with an intermediate shade of grey, and the figure illustrates how this layer is eroded with time. The intruding layer is also shaded in light grey. We have also calculated the evolution of a purely draining flow with no plume heat source for $\tau>0$, by solving the equation for $\zeta_{o}$ in (2.14) with $q_{e}=0$ and $q_{t}$ determined using $\hat{g}_{i}=0$ in (2.8). The result of this calculation is plotted with a dashed line.

In this transient flow, the total depth of the buoyant layers decreases transiently as expected when the source buoyancy flux is decreased: this is a result of the reduced volume flux of the plume following the reduction in the heat load. Since the upper layer initially drives a ventilation flow with volume flux equal to that of the original plume at the level of the interface, then with the reduced intensity of the plume, the interface rises in order to match the ventilation flow. The figure also shows that the original layer disappears more rapidly than in the pure draining flow in which the heat source is set to zero (as plotted with the dashed line). This is because of the warm intruding layer associated with the continuing heat source which, relative to the case of removing the heat source, increases the net buoyancy of the column of air in the room and hence the ventilation flow.

The flow is sensitive in two ways to the particular choice of the entrainment parameter $E$ within our simple model, as shown in figure $3(c)$, in which the evolution of the height of the original interface $\zeta_{o}(\tau)$ is plotted (with thick lines) and the intruding interface $\zeta_{i}(\tau)$ (with thin lines) for four different choices of $E$. The case $E=0$ corresponds to us assuming within our model that the flow has no entrainment. As we increase $E$ within our model over the range $0<E<1$, the model predicts a more rapid depletion of the original warm layer. The particular chosen value of $E$ has much less impact on the predicted height of the new intruding interface at $\zeta_{i}$. While the original layer persists, the total buoyancy of the plume fluid in the room is not affected by penetrative entrainment, as the penetrative entrainment merely acts to redistribute buoyancy between the two layers. Therefore, the flow $q_{t}$ through the top opening is unaffected by the entrainment of original fluid into the intruding layer, until the original layer is completely depleted. Subsequently however, there is a slight variation, since the reduced gravity of the fluid that is leaving through the top opening varies depending on the value of the entrainment parameter, as this will modify the reduced gravity of the remaining intruding layer. (We discuss this point further in $\S 3$ below.)

The other marked effect of penetrative entrainment is in variation of the reduced gravity of the intruding layer. In figure $3(d)$, the model prediction of the reduced gravity of the new intruding layer is plotted as a fraction of the buoyancy of the original layer, $\hat{g}_{i} / \hat{g}_{o}$ for the various values of $E$. For reference, for the particular flow considered here $\hat{g}_{o}=2^{-5 / 3}=0.32$, and $\hat{g}_{i} / \hat{g}_{o} \rightarrow f_{-}^{2 / 3}=0.34$ at long times. When 
there is no penetrative entrainment, the reduced gravity of the newly intruding layer initially decreases as the interface $\zeta_{i}$ recedes upwards, and so the incoming plume fluid becomes relatively more diluted by entrained ambient fluid. Subsequently, the buoyancy increases again as the layer relaxes back to its asymptotic value. If there is any pentrative entrainment, the initial entrainment of less dense fluid tends to increase the initial reduced gravity of the intruding layer, as is apparent on figure $3(d)$. Indeed, for a sufficiently large value of $E$ in the model, the initial penetrative entrainment of original layer (and hence less dense) fluid increases the reduced gravity sufficiently to dominate the initial reduction of the reduced gravity of the incoming plume fluid due to the transient decrease of the total layer depth, and so $\hat{g}_{i}$ starts from a high value, which then decreases monotonically with time.

It is important to appreciate that this simplified entrainment model, and in particular the assumption that $E$ is independent of the magnitude of the change in heating, $f_{-}$, or the height of the steady-state interface, $\zeta_{\infty}$, is only likely to be appropriate for intermediate values of decreased buoyancy flux $f_{-}$. If $f_{-}$is close to one, the penetrative entrainment is likely to be so intense that the plume may rise to the top of the space and no clear intruding layer will form. Conversely, if $f_{-}$is sufficiently small, then the plume fluid may be so dense that penetrative entrainment is suppressed. In particular, care must be taken in drawing conclusions from empirical determinations of the parameter $E$, outside the range of $f_{-}$and $\zeta_{\infty}$ covered by our laboratory experiments. Furthermore, the assumption that the intruding layer is always thoroughly well mixed when there is penetrative entrainment is always going to be a simplification (though perhaps a useful one), at least at early times. Indeed, empirical determination of the entrainment parameter must be assessed critically, as interface location measurements may well imply a different value for $E$ from that which is implied by measurements of the density in the intruding layer. We test the usefulness of our model in the next section with a series of experimental measurements, paying particular attention to circumstances testing the underlying assumptions of our model.

\section{Experiments}

\subsection{Experimental method}

We conducted a systematic series of small-scale analogue laboratory experiments to test our models. The experiments were conducted in a model room, which was immersed, upside down, in a tank of fresh water. Localized heat sources were then modelled by sources of brine, exploiting the Boussinesq approximation (Linden 1999). The model room was constructed from acrylic sheet, with internal dimensions $0.316 \times$ $0.316 \times 0.372 \mathrm{~m}$. Five circular outflow openings with diameter $15 \mathrm{~mm}$ could be opened at the base of the walls, while the upper part of the room was completely open. Using this approach, $A_{\star} \simeq \sqrt{2} c_{d} A_{o}$, where $A_{o}$ is the total area of the unplugged openings, and $c_{d}$ is the appropriate discharge coefficient. For this experimental geometry, Gladstone \& Woods (2001) have determined that $\sqrt{2} c_{d} \simeq 0.98$, a number which we verified by a sequence of simple draining experiments. The model room was submerged in a reservoir tank of fresh water of dimensions $2.00 \times 0.74 \times 0.76 \mathrm{~m}$, which, over the time scale of the experiments, provided an unchanging uniform environment.

Two turbulent plume sources were positioned side-by-side at the centre of the building model, with the exit nozzles approximately $1 \mathrm{~cm}$ below the top level of the model. Each plume source was supplied with a saline solution (ranging between $2 \%$ and $20 \% \mathrm{NaCl}$ by weight) thus allowing the system to experience an instantaneous 
change in buoyancy at the source. The source volume flux was calibrated through direct measurement. The original source was typically used for a sufficiently long time for both the depth and density of the original layer to be close to its steady-state value. The time $\tau=0$ was then chosen to correspond to the instant at which the new plume material first reaches the steady-state interface height.

As discussed in Woods, Caulfield \& Phillips (2003), with a finite source mass flux, the steady-state interface location depends on the source conditions. In general, the interface height is a function of the source momentum flux, volume flux and buoyancy flux. Provided the steady-state location of the interface is sufficiently far from the source, however, differences in the steady interface location for plumes of different source conditions are principally dependent on the virtual origin from which the plume appears to be rising, $z_{v}$. The location $z_{v}$ is such that $Q=\lambda F_{s}^{1 / 3}\left(z+z_{v}\right)^{5 / 3}$ sufficiently far from the source, with $\lambda$ as defined in (2.2) (Caulfield \& Woods 1995; Hunt \& Kaye 2001). The distance of the virtual origin behind the actual source scales with $b_{s}$, the source dimension, but also depends on the dimensionless source parameter $\Gamma$ (Morton 1959) given by

$$
\Gamma=\frac{5 Q_{s}^{2} F_{s}}{4 \alpha M_{s}^{5 / 2}}=\frac{5 \pi^{5 / 2} b_{s}^{5} g_{s}^{\prime}}{4 \alpha Q_{s}^{2}} .
$$

In order that the strong and weak plumes have close to the same interface height, so that the final steady interface matches the initial steady interface, the two plume sources require the same virtual origin. The experimental sources we used were based on the design of Dr Paul Cooper (see Hunt \& Linden 2001), and have a nearly uniform source velocity across the source radius. Therefore $M_{s} \sim Q_{s}^{2} /\left(\pi b_{s}^{2}\right)$ and so in order that two plumes have the same value of $\Gamma$, we require that $Q_{1}^{2} / g_{1}^{\prime}=Q_{2}^{2} / g_{2}^{\prime}$. This quantity was therefore always matched in our experiments as the buoyancy flux was changed.

Steady state, with interface location $\zeta=\zeta_{s s}$ occurs when the plume volume flux into the buoyant layer $q_{p}\left(\zeta_{s s}\right)$ exactly matches the ventilation flow, $q_{t}$, through the top opening. Although the sources had non-zero source volume flux, since the tank is open at the top, and so the hydrostatic pressure is equal inside and outside there, the outflow $q_{t}$ is still given by (2.6) and (2.8) for the filling flow in which the buoyancy flux is increased and the intruding flow in which the buoyancy flux is decreased. Therefore, at steady state,

$$
q_{t}^{3}=\frac{\zeta_{\infty}^{5}\left(1-\zeta_{s s}\right)}{\left(1-\zeta_{\infty}\right)}=q_{p}^{3}\left(\zeta_{s s}\right),
$$

where $q_{p}\left(\zeta_{s s}\right)$ is determined as a solution of the plume equations (2.5). In solving the plume equations, we have used the initial conditions at $z=0$ that $q=q_{s}, m=m_{s}$, and $f=f_{-}$. It is important to appreciate that $\zeta_{s s}<\zeta_{\infty}$, since finite source volume flux reduces the interface location compared to the point source value $\zeta_{\infty}$ (see Woods et al. 2003 for more details).

Considering (3.2), it is clear that the value of the entrainment constant $\alpha$ appears in two places: in the scaling of the source conditions defined by (2.3) and the scaling of $\zeta_{\infty}$ from (2.9), where as already noted, the appropriate value of the discharge coefficient can be determined completely independently through draining experiments. Therefore, for any given value of $\alpha$, (3.2) implicitly defines a value of $\zeta_{s s}$ which can be compared with experimental measurement. We conducted 47 different experiments which we allowed to evolve to steady state. The most consistent choice of $\alpha$ with this data has value $\alpha=(0.096 \pm 0.007) \sqrt{2}$ (equivalently, $\lambda=0.17$ as defined in (2.2), when 
the velocity and density distributions were assumed to be 'top-hat' for simplicity). This choice of $\alpha$ implied an r.m.s. error between (3.2) and the experimental measurements of less than $4 \mathrm{~mm}$, which is within the precision of our experimental measurement techniques. This technique follows the approach of Baines (1975) for determining $\alpha$.

The other parameter which needs to be empirically determined is the entrainment parameter $E$ as defined implicitly in (2.16). To determine this, we conducted eight intruding flow experiments, varying both the ratio of the source buoyancy fluxes (and hence $f_{-}$) and the opening area (and hence $\zeta_{\infty}$ ). For this sequence of experiments, the principal experimental measurement was the time evolution of the interface height. These measurements, with a precision of $\sim 5 \mathrm{~mm}$, were taken using the shadowgraph technique, where the light source was sufficiently far from the tank for any parallax errors to be extremely small. It is important to appreciate that this method identifies variation of light intensity proportional to the Laplacian (here dominated by the second $z$-derivative) of the refractive index, and hence the density of the fluid (see Settles 2001 for more detail). Therefore, the interface identified will correspond to the location where the density gradient is most rapidly varying. If the intruding layer is not completely well mixed, this may well not correspond to alternative reasonable definitions of the interface between the two regions of fluid, for example the midpoint of the region over which density varies significantly.

For a given value of $E$, we solved the model system (2.14) for each of the eight intruding-flow experimental data sets. We then compared the model predictions with the measurements of both the interface locations which were taken at each of the 100 time intervals during each experiment, as the flow evolved. We have found that the r.m.s. error between the theoretical predictions and the experimental measurements of the interface locations can be minimized and takes the value $5-6 \mathrm{~mm}$, when $E=1.06$. However, we observed that the models are only weakly sensitive to the precise value of $E$, and the experimental interface location data are consistent (to within an r.m.s. error of less than $1 \mathrm{~cm}$ ) with the model predictions using $E$ in the range $0.5<E<1.5$. This is in accord with the value $E=0.65$ reported by Lin \& Linden (2005) (i.e. $E=0.65 \pm 0.17$ ).

It is important to appreciate that we considered only intermediate values $0.1<f_{-}<0.4$ and so caution should be exercised in extrapolating outside that range of values. Experimental issues make it extremely difficult to consider a wider range of $f_{-}$. The largest possible buoyancy flux is constrained by three principal requirements. Firstly, the models using salt constrains the largest possible density difference to be of the order of $15 \%$, just on the limit of consistency with the Boussinesq approximation. Secondly, the source momentum flux must be sufficiently small to ensure that the whole space does not overturn (Baines \& Turner 1969). Thirdly, the source volume flux must be sufficiently small to ensure that the flow remains naturally ventilated (Woods et al. 2003). If the reduced buoyancy flux is too close to the original buoyancy flux, it is extremely difficult to detect an intruding interface, thus constraining the largest possible accessible value of $f_{-}$, while very small values of source buoyancy flux typically do not exhibit turbulent plume-like behaviour on the laboratory scale, thus constraining the smallest possible accessible value of $f_{-}$. Nevertheless, we believe that experiments covering this range of $f_{-}$allow us to consider the fundamental flow dynamics of such intruding flows.

\subsection{Experimental observations}

We show images from an illustrative filling flow experiment in figure 4. The two sources at the top of the tank are clearly visible. The openings to the reservoir tank 


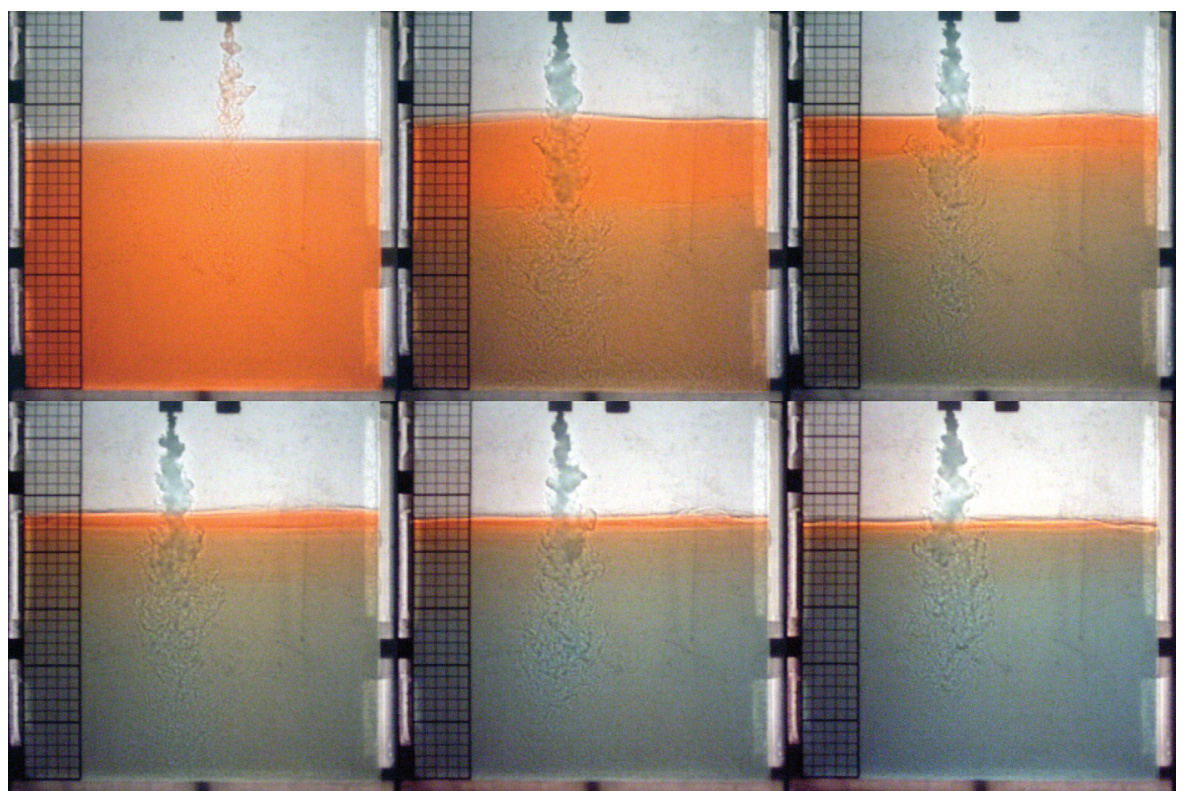

FiguRE 4. Experimental images for a filling flow with $\zeta_{\infty}=0.39$ and $f_{+}=8.5$ at $\tau=<0 ; 0.5 ; 1$; $1.5 ; 2 ; 2.5$. The original (less dense) plume fluid is coloured red and the final (more dense) plume fluid is coloured blue. Each square is $1 \mathrm{~cm}^{2}$. The source has dimensional radius $b_{s}=2.5 \mathrm{~mm}$. The initial dimensional source volume flux $Q_{o}=0.40 \mathrm{~cm}^{3} \mathrm{~s}^{-1}$ and the final dimensional source volume flux $Q_{+}=0.79 \mathrm{~cm}^{3} \mathrm{~s}^{-1}$. The initial dimensional source buoyancy flux $F_{o}=0.14 \mathrm{~cm}^{4} \mathrm{~s}^{-3}$ and the final dimensional source buoyancy flux $F_{+}=1.2 \mathrm{~cm}^{4} \mathrm{~s}^{-3}$.

are in the bottom right corner of each image. For scale, the squares on the left are $1 \mathrm{~cm}^{2}$ each. For this experiment, $\zeta_{\infty}=0.39$ and $f_{+}=8.5$. The original plume (from the right-hand source) is coloured red, while the more dense filling plume is coloured blue. The images were taken at 2-min intervals, corresponding to dimensionless times $\tau<0$ (approaching the original steady state), $\tau=0.5, \tau=1, \tau=1.5, \tau=2$ and $\tau=2.5$. The characteristic transient deepening of the total depth of the two layers is apparent due to the increased volume flux associated with the denser plume. Also, the more dense plume completely penetrates the original layer, and fills the room from below. The filling layer is purple in colour due to the entrainment by the more dense plume as it passes through the original layer.

The dynamics are qualitatively different in the illustrative intruding flow experiment images shown in figure 5. As in figure 4, the less dense plume is coloured red, and the more dense plume is coloured blue, and for this experiment the dimensionless times shown are $\tau<0$ (approaching the original steady state), $\tau=1, \tau=2, \tau=3, \tau=4$ and $\tau=5$. (These correspond once again to 2-min intervals of real time, but since the initial buoyancy flux is larger in this case by a factor of eight, the filling box time $T_{o}$ defined using the original buoyancy flux as in (2.4) is smaller by a factor of two.) The characteristic transient reduction in total depth of the two layers is apparent due to the reduced volume flux of the less dense plume. The intrusion of the plume fluid can also be seen, and there is clear visual evidence of the overshoot of the plume into the original layer. Some penetrative entrainment appears to be occurring, even at quite late times (e.g. $\tau=3$ ). Essentially, these illustrative experiments exhibit very similar dynamics to the schematic flows presented in figure 1, allowing of course for the 


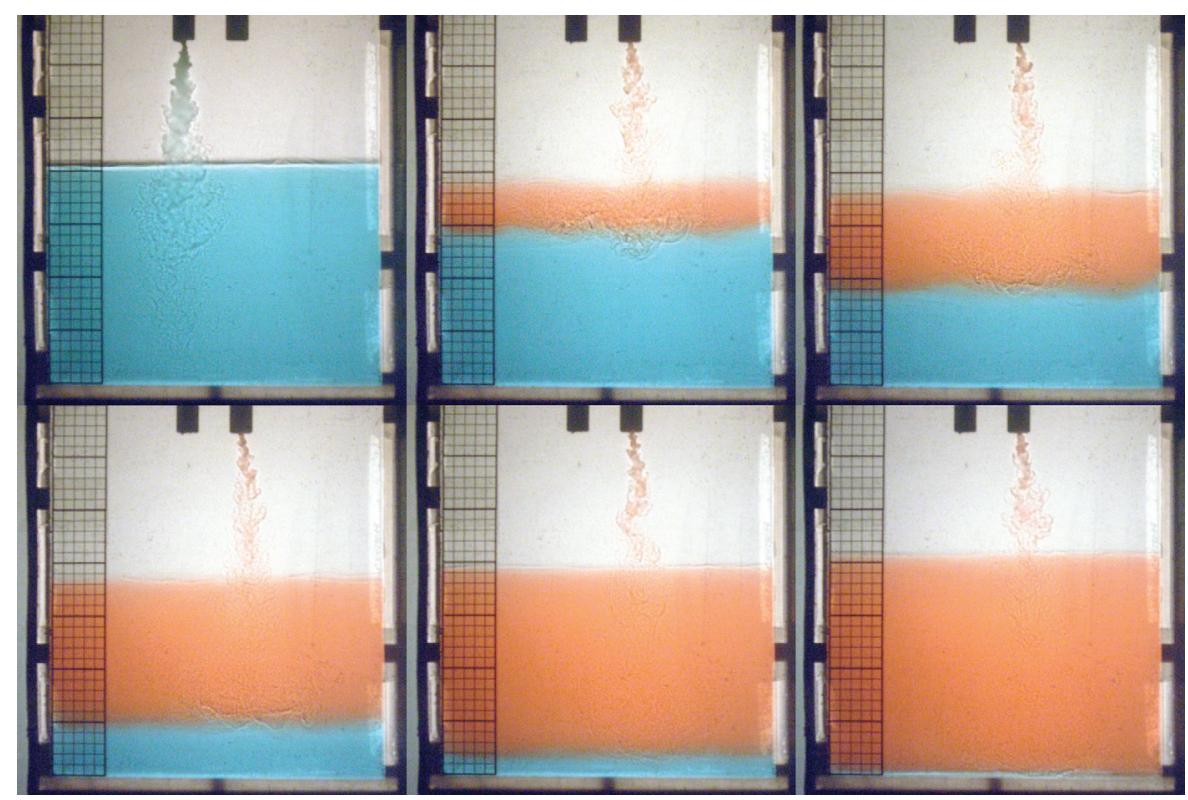

FIGURE 5. Experimental images for an intruding flow with $\zeta_{\infty}=0.39$ and $f_{-}=0.12$ at $\tau=<0$; $1 ; 2 ; 3 ; 4 ; 5$. The original (more dense) plume fluid is coloured blue and the final (less dense) plume fluid is coloured red. Each square is $1 \mathrm{~cm}^{2}$. The thin layer of original fluid visible in panel f remains for a long time due to the finite height of the openings to the external reservoir. The source has dimensional radius $b_{s}=2.5 \mathrm{~mm}$, The initial dimensional source volume flux $Q_{o}=0.79 \mathrm{~cm}^{3} \mathrm{~s}^{-1}$ and the final dimensional source volume flux $Q_{-}=0.40 \mathrm{~cm}^{3} \mathrm{~s}^{-1}$. The initial dimensional source buoyancy flux $F_{o}=1.2 \mathrm{~cm}^{4} \mathrm{~s}^{-3}$ and the final dimensional source buoyancy flux $F_{-}=0.14 \mathrm{~cm}^{4} \mathrm{~s}^{-3}$.

vertical inversion due to the use of sources of brine to model heat sources, and they present some evidence that it is sensible to model the flow with a non-zero value of $E$.

\subsection{Profile evolution}

To investigate whether our models are useful to describe experimental flows quantitatively, we conducted three filling flow experiments and three intruding flow experiments. We varied both the ratio of the initial and final buoyancy fluxes, and the effective opening areas. For the intruding flows, the parameter values chosen were very similar to those used to determine the empirical value of $E$. We measured vertical profiles of the density using a conductivity probe approximately every minute. The measured conductivity was calibrated with both salt concentration and temperature. The temperature of the fluid was carefully controlled, as the typical salt concentrations in the intruding layer were of the order of $0.1 \%$, and so thermal variation in density could be significant. Profiles of dimensionless reduced gravity $\hat{g}$ (as defined in (2.3)) are plotted with a thick solid line in figure 6 for the filling flow experiments and in figure 7 for the intruding flow experiments. In each case, the profiles are offset by a constant amount ( 40 for the filling flows and 10 for the intruding flows) for clarity. For the sake of comparison, profiles plotted in figure 6(b) were measured in an experiment with very similar parameter values to the experiment shown in figure 4 , while profiles plotted in figure $7(b)$ were measured in an experiment with very similar parameter values to the experiment shown in figure 5.

In figure 6, the theoretical prediction of the evolution of the reduced gravity in each layer is also plotted with a thin solid line. In figure 7, the evolution of the theoretical 

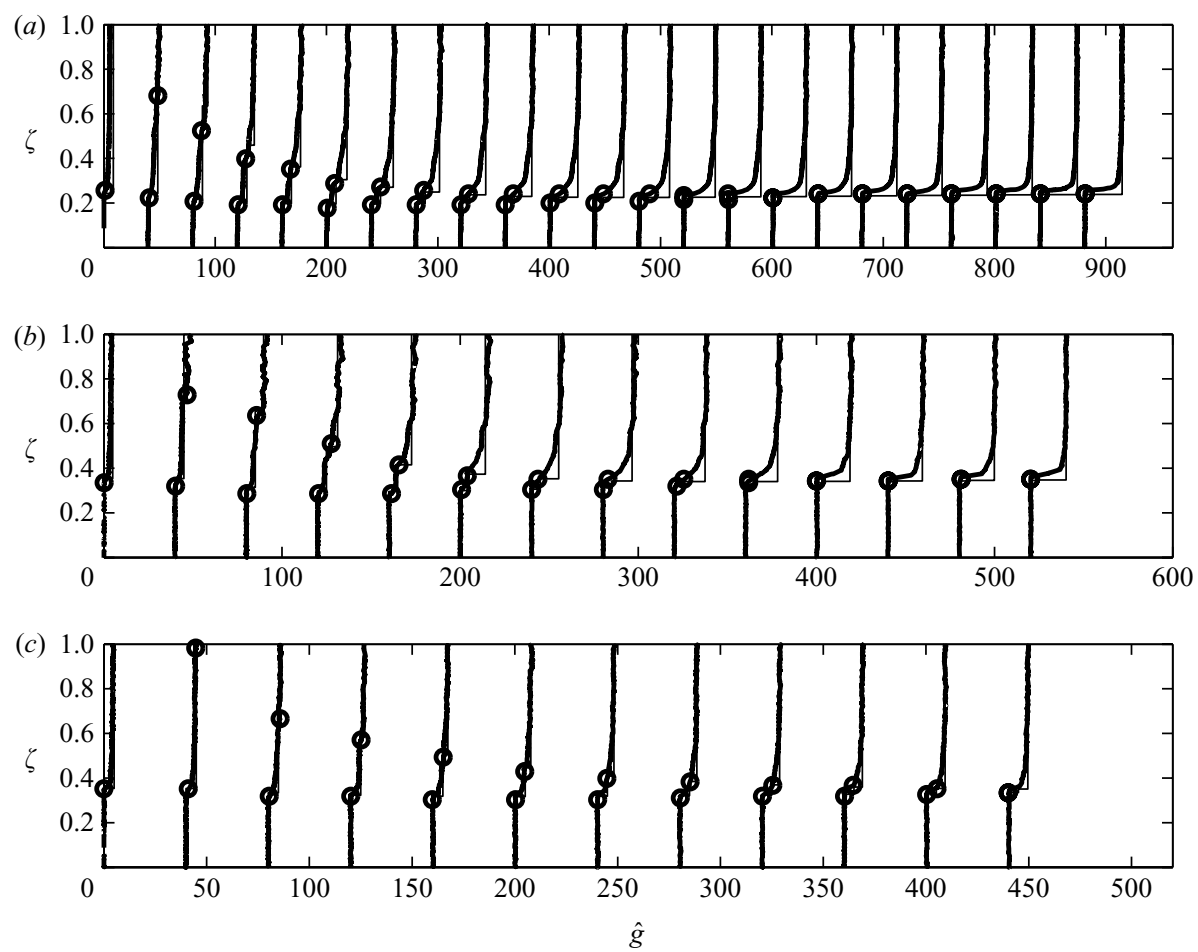

FIGURE 6. Experimental measurements of reduced gravity profiles $\hat{g}(\zeta)$ (plotted with thick solid lines, and offset by 40 units for each profile) for the filling flow experiments with $(a) \zeta_{\infty}=0.27$, $f_{+}=8.7$, final dimensional source volume flux $Q_{+}=0.78 \mathrm{~cm}^{3} \mathrm{~s}^{-1}$ and final dimensional source buoyancy flux $F_{+}=1.1 \mathrm{~cm}^{4} \mathrm{~s}^{-3}$ at times $\tau<0, \tau=0.19,0.47,0.75,1.0,1.3,1.6,1.9,2.1$, $2.4,2.7,3.0,3.2,3.5,3.8,4.1,4.3,4.6,4.9,5.2,5.5,5.7,6.0 ;(b) \zeta_{\infty}=0.38, f_{+}=8.7$, final dimensional source volume flux $Q_{+}=0.78 \mathrm{~cm}^{3} \mathrm{~s}^{-1}$ and final dimensional source buoyancy flux $F_{+}=1.1 \mathrm{~cm}^{4} \mathrm{~s}^{-3}$ at times $\tau<0, \tau=0.1,0.38,0.66,0.93,1.2,1.5,1.8,2.0,2.3,2.6,2.9,3.1$, $3.4 ;$ (c) $\zeta_{\infty}=0.38, f_{+}=3.1$, final dimensional source volume flux $Q_{+}=0.55 \mathrm{~cm}^{3} \mathrm{~s}^{-1}$ and final dimensional source buoyancy flux $F_{+}=0.41 \mathrm{~cm}^{4} \mathrm{~s}^{-3}$ at times $\tau<0, \tau=0.04,0.32,0.60,0.88$, $1.2,1.4,1.7,2.0,2.3,2.6,2.8$. For all experiments, the source radius $b_{s}=2.5 \mathrm{~mm}$, the initial dimensional source volume flux $Q_{o}=0.38 \mathrm{~cm}^{3} \mathrm{~s}^{-1}$ and the initial dimensional source buoyancy flux $F_{o}=0.13 \mathrm{~cm}^{4} \mathrm{~s}^{-3}$. The theoretical predictions for the reduced gravity of each well-mixed layer and their locations are plotted with a thin solid line. Visual measurements of the interface locations are plotted with open circles.

prediction for the reduced gravity with penetrative entrainment $(E=1.06)$ is plotted with a thin solid line, while the theoretical prediction with no penetrative entrainment (i.e. with $E=0$ ) is plotted with a dashed line. On all figures, the visually identified interface locations (using the shadowgraph technique) are plotted with open circles.

Considering the filling flow profiles first, as expected the interface locations are well predicted. As in figure $3(a)$, the new filling layer deepens in a monotonic manner towards its steady state. This deepening is somewhat faster when $f_{+} \gg 1$ (cf. the fifth profiles on figures $6(b)$ and $6(c)$ around $\tau=0.9$, slightly above 150$)$ as the increased buoyancy flux implies larger volume fluxes. The presence of the original layer delays the approach of the filling layer towards its final steady state due to the greater value of $q_{t}$ associated with the enhanced buoyancy in the two layers. This difference is relatively small however, and it seems that the appropriate time for the filling flow evolution is the filling box time scale associated with the increased buoyancy flux $f_{+}$. 

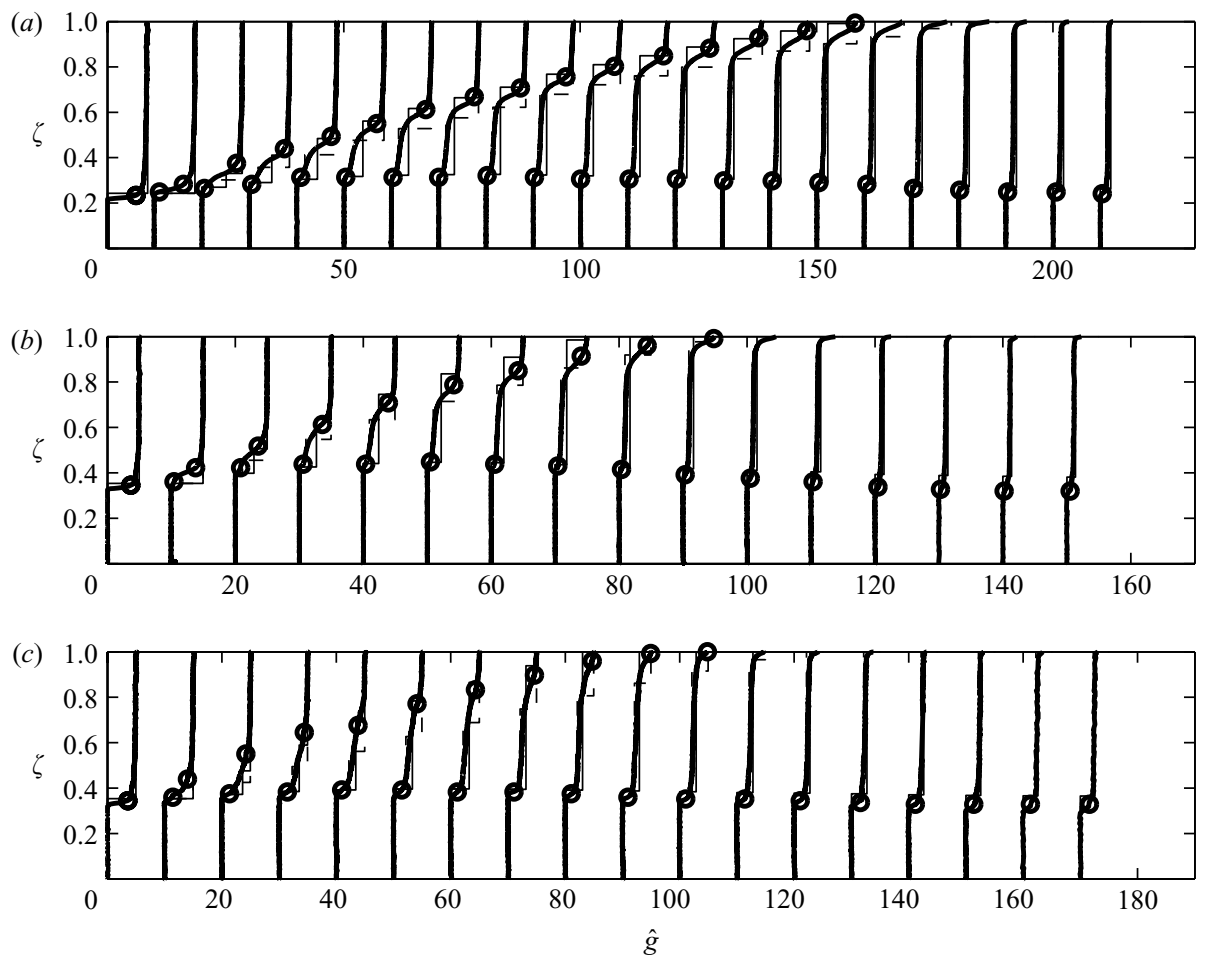

FIGURE 7. Experimental measurements of reduced gravity profiles $\hat{g}(\zeta)$ (plotted with thick solid lines, and offset by 10 units for each profile) for the intruding flow experiments with (a) $\zeta_{\infty}=0.27, f_{-}=0.12$, initial dimensional source volume flux $Q_{o}=0.78 \mathrm{~cm}^{3} \mathrm{~s}^{-1}$ and initial dimensional source buoyancy flux $F_{o}=1.1 \mathrm{~cm}^{4} \mathrm{~s}^{-3}$ at times $\tau<0, \tau=0.42,0.94,1.5,2.0,2.6,3.2$, $3.7,4.2,4.9,5.5,6.0,6.5,7.1,7.6,8.1,8.7,9.3,9.9,10.4,11.0,11.5 ;(b) \zeta_{\infty}=0.38, f_{-}=0.12$, initial dimensional source volume flux $Q_{o}=0.78 \mathrm{~cm}^{3} \mathrm{~s}^{-1}$ and initial dimensional source buoyancy flux $F_{o}=1.1 \mathrm{~cm}^{4} \mathrm{~s}^{-3}$ at times $\tau<0, \tau=0.36,0.88,1.4,1.9,2.5,3.0,3.6,4.1,4.7,5.3,5.8,6.8,7.4$, $7.9,8.4 ;(c) \zeta_{\infty}=0.38, f_{-}=0.34$, initial dimensional source volume flux $Q_{o}=0.54 \mathrm{~cm}^{3} \mathrm{~s}^{-1}$ and initial dimensional source buoyancy flux $F_{o}=0.40 \mathrm{~cm}^{4} \mathrm{~s}^{-3}$ at times $\tau<0, \tau=0.28,0.64,1.0$, $1.4,1.8,2.1,2.5,2.9,3.2,3.6,4.0,4.4,4.7,5.5,5.8,6.2,6.6$. For all experiments the source radius $b_{s}=2.5 \mathrm{~mm}$, the final dimensional source volume flux $Q_{-}=0.39 \mathrm{~cm}^{3} \mathrm{~s}^{-1}$ and the final dimensional source buoyancy flux $F_{-}=0.13 \mathrm{~cm}^{4} \mathrm{~s}^{-3}$. Visual measurements of the interface locations are plotted with open circles. The theoretical predictions for the reduced gravity of each well-mixed layer and their locations are plotted with a thin solid line when there is assumed to be penetrative entrainment (with entrainment parameter $E=1.06$ ) and with a dashed line when there is assumed to be no penetrative entrainment (i.e. $E=0$ ).

Nevertheless, there is a consistent bias in the visual measurements towards the base of the filling layer. This is unsurprising as it is the transition region of the most rapid change of density gradient from the fresh layer to the salty layer. The erosion of the original layer is captured clearly by the density profile. Furthermore, the density of the original layer is close to uniform (in the sense that the density variations within the initial layer are substantially smaller than the density variation between the final filling layer and the ambient fluid) and captured by the emptying filling box model, appropriately modified to capture the effect of finite source volume flux (Woods et al. 2003). The density of the filling layer is not uniform however, but has a very similar structure to the profile considered by Caulfield \& Woods (2002) for a finite volume flux emptying filling box. This is unsurprising, since on entering the filling layer, the 
plume has significant volume flux. As discussed in Caulfield \& Woods (2002), such a layer evolves towards uniform density, and the dynamics are typically little affected by the non-uniformity in the density profile. Also, it is clear that the well-mixed layer predicts a density very close to that of the average value of the density of the actual layer, and so the well-mixed assumption appears thoroughly reasonable.

Turning attention to the intruding flow profiles, the situation is substantially more complicated. Certain characteristics are apparent. The reduction in total depth of the buoyant layers is observed, and agrees well with the models for all three experiments. The density of the intermediate layer throughout much of its depth is typically close to well mixed, although there is some evidence of a gradient throughout much of the layer (for example the 11th profile for $\tau=5.5$ around 100 in figure $7 a$ ), and there is typically a thin region of relatively strong gradient at the top of the layer. This region of strong gradient may be thought of as being caused by penetrative entrainment by the intruding plume at the early stages of the intermediate layer development. Also, the visual measurement of the original interface location better matches the model with penetrative entrainment with $E=1.06$ (plotted with a solid line) than the non-entraining model (plotted with a dashed line), particularly for the cases shown in figures $7(a)$ and $7(c)$.

Indeed, when the buoyant layers are sufficiently shallow, and the density change is sufficiently large (i.e. figure $7 b$ ) penetrative entrainment is relatively unimportant. However, when the layers are deep (i.e. figure $7 a$ ) or particularly when the buoyancy flux reduction is relatively small (i.e. figure $7 c$ ) the penetrative entrainment plays a significant role in the reduction of the original layer depth. Also, the experiments suggest that the flow evolves on the draining time scale associated with the original buoyant layer rather than the filling box time, as is apparent through the comparison between the thick solid line and the dashed line.

However, the detailed observation of the density profile shows some discrepancies between the entraining model and the observations. These discrepancies are masked by the visual observations of the density interface locations. The real behaviour of the system lies somewhere between the non-entraining and entraining models' behaviour, and these two models should be considered as end-members bracketing the actual flow behaviour. Most significantly, in general the reduced gravity of the intruding layer is very close to the reduced gravity predicted by the non-entraining model, with a relatively thin region (of the order of $1-2 \mathrm{~cm}$ ) of varying reduced gravity at the top of the intruding layer. This is not entirely surprising, as the penetrative entrainment is unlikely to lead to a completely well-mixed intruding layer, particularly when the total intruding layer is relatively deep. Since the entrained fluid is more buoyant than the intruding fluid, it is likely to remain above the intruding layer.

Furthermore, the visual measurements of the interface location tend to correspond, as in the filling flows, with the edge of the transition region between the layers, where the density gradient varies most rapidly. This bias, particularly at the transition region from the original layer to the intruding layer, tends to make the agreement with the entraining model appear somewhat better than it actually is at predicting the behaviour of the system.

\subsection{Interface measurement}

We analysed interface measurements from a sequence of fourteen transient experiments, including six filling experiments with increased buoyancy flux and the eight intruding experiments with decreased buoyancy flux used to determine an empirical value for $E$. We used six different groups of experimental parameters, 

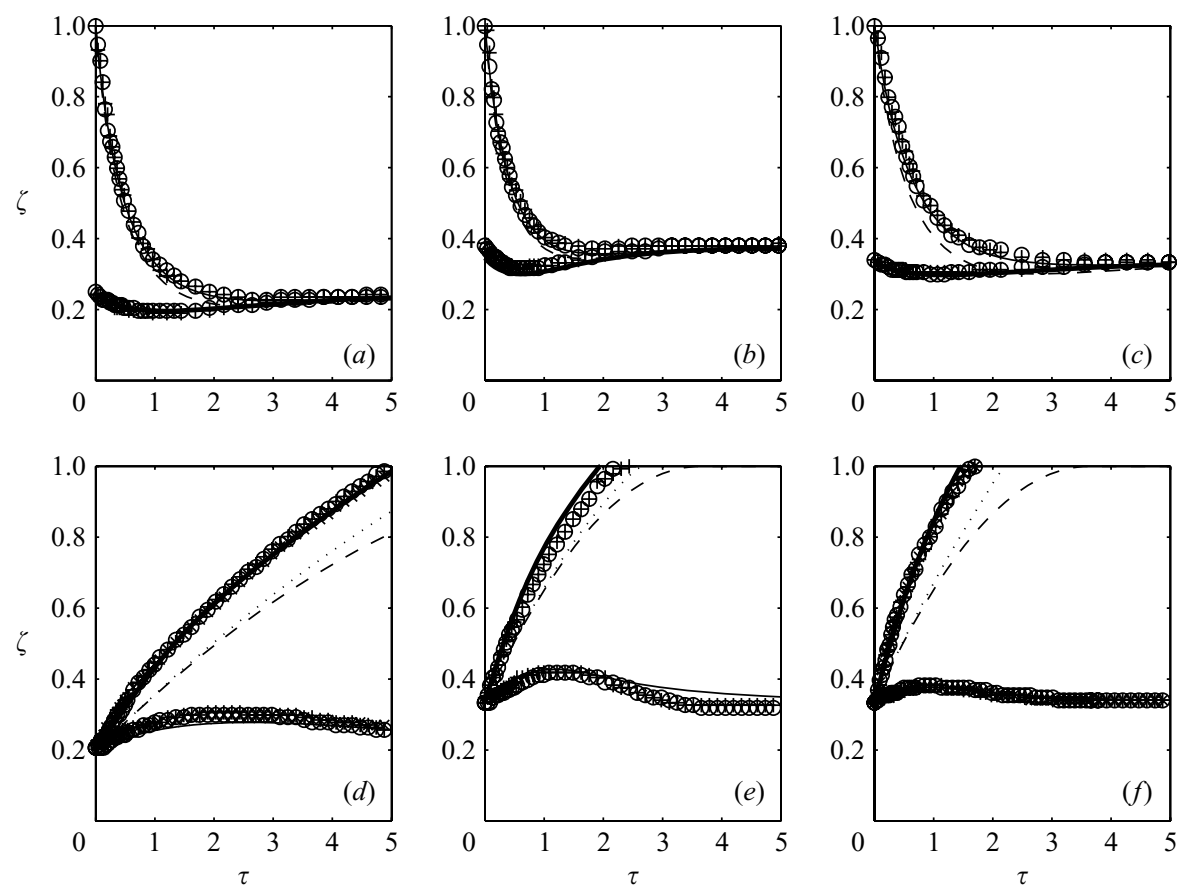

FIGURE 8. Experimental measurements (plotted with symbols) and theoretical predictions for the original interface (thick solid lines) and either the filling $(a-c)$ or intruding $(d-f)$ interface (thin solid lines) plotted against dimensionless time $\tau$ for experiments with: $(a)$ $\zeta_{\infty}=0.27, f_{+}=8.0 ;(b) \zeta_{\infty}=0.40, f_{+}=8.4 ;(c) \zeta_{\infty}=0.36, f_{+}=3.0 ;(d) \zeta_{\infty}=0.26, f_{-}=0.12$; (e) $\zeta_{\infty}=0.36, f_{-}=0.12 ;(f) \zeta_{\infty}=0.36, f_{-}=0.36$. Different symbols correspond to different experiments with the same parameters. The steady-state interface locations naturally differ from $\zeta_{\infty}$ due to the finite volume flux at the source. In $(a-c)$, the evolution of the interface which develops when a plume with buoyancy flux $f_{+}$fills a room initially containing only ambient fluid is also plotted (with a dashed line). In $(d-f)$, the evolution of the original interface if it drains with no plume is plotted with a dashed line and the evolution of the interfaces are plotted with dotted lines if there is no penetrative entrainment at all, and so $q_{e}=0$.

closely related to the values used in the six experiments whose results are shown in figures 6 and 7. Every combination of external opening area and original and final source density was used at least twice. This verified that the results were reproducible.

For filling flows, the source buoyancy flux was increased, $f_{+}>1$. Three different combinations of opening areas and source buoyancy fluxes are shown in figure $8(a-c)$. For comparison, the parameters for the experimental results shown in figure $8(b)$ are similar to those for the illustrative experiment shown in figure 4, while the parameters for the experimental results shown in figure $8(a-c)$ correspond closely to those for the experimental results shown in figure $6(a-c)$ respectively.

In figure $8(d-f)$, we present the results of the eight different experiments we conducted for intruding flows in which the source buoyancy flux is decreased. Once again, experimental data are marked with symbols, while the theoretical predictions of the height of the original and intruding interfaces are marked with thick and thin solid lines respectively. For comparison, the parameters for the experimental results shown in figure $8(e)$ are similar to those for the illustrative experiment shown in 
figure 5, while the parameters for the experimental results shown in figure $8(d-f)$ are similar to those for the experimental results shown in figure $7(a-c)$, respectively.

The model predictions for the evolution of the two interfaces are in good accord with the experimental data. In particular the model of penetrative entrainment is adequate to describe the enhanced erosion of the original interface compared to the flow with no entrainment (by comparison with the predicted evolution of the flow when $E=0$, as plotted with a dotted line), at least for flows with intermediate values of $f_{-}$and $\zeta_{\infty}$, or equivalently $\zeta_{s s}$. This is not entirely surprising, as we used the data from these experiments to determine the most appropriate value of the entrainment parameter $E$.

We note that the penetrative entrainment is overestimated by the simplified model herein when the change in the buoyancy flux is relatively large and the plume has had a relatively large distance to propagate. In this case, the velocity in the plume decreases substantially as it migrates through the intruding layer towards the upper interface (figure $8 e$, which is a similar experiment to those shown in 5 and $7 b$ ). This overestimate of the entrainment leads to the prediction of a more rapid erosion of the upper original layer than observed in the experiments.

It may therefore be appropriate to model the entrainment coefficient $E$ as $E(F r)$, as postulated by Linden (1973), Baines (1975), Kumagai (1984), where $F r$ is the interfacial Froude number defined as

$$
F r=\frac{w\left(\zeta_{o}\right)}{\left[b\left(\zeta_{o}\right)\left(g / \rho_{a}\right)\left(\rho\left(\zeta_{o}\right)-\rho_{o}\right)\right]^{1 / 2}}
$$

This quantifies the relative strength of the density jump at the original layer compared to the arriving plume's inertia. However, we do not believe such added complexity substantially improves the quality of the model as a predictive and descriptive tool. Indeed, both the entraining and non-entraining models capture different aspects of the flow evolution, and the non-entraining model is useful to capture the bulk properties of the intruding layer, particularly when the change in reduced gravity and the steady-state depth of the layer is relatively large (e.g. for the flow parameters of the experimental results shown in figures $5,7 b$ and $8 e$ ).

\section{Conclusions}

In this paper, we have considered the transient evolution of an emptying filling box when the source buoyancy flux is changed instantaneously. There are two qualitatively different flows which may develop, depending on whether the source buoyancy flux is increased or decreased after the original flow has reached steady state, with a well-mixed buoyant layer in the upper part of the room. If the source buoyancy flux is increased, we refer to the flow as a 'filling' flow, since the more buoyant continuing plume rises through the original layer, and fills the room from the ceiling downwards. The original layer is eroded by entrainment into this plume as it rises, and a new buoyant layer fills the room from the top. Typically, the total depth of the buoyant layer overshoots both its original and final values. The flow evolves on the natural filling box time scale of the enhanced buoyancy flux plume, and there is very good agreement between simple models based on the evolution of well-mixed layers, and the results of a range of laboratory experiments.

On the other hand, if the source buoyancy flux is decreased, we refer to the flow as an 'intruding' flow, and the flow dynamics are qualitatively different. The plume now becomes a dense fountain as it intrudes into the initial buoyant layer, and 
then collapses back to form an intruding layer below the original layer. The original layer reduces in depth in two ways: drainage through the top opening and penetrative entrainment by the fountain. The combined depth of the buoyant layers actually decreases transiently, since the volume flux draining through the top opening is typically larger than the volume flux in the plume with decreased buoyancy flux as it enters the buoyant layers. The time scale of evolution of this flow appears to be related to, but to be somewhat faster than the time scale of the draining flow associated with the original buoyant layer.

Penetrative entrainment is less important when the reduction in buoyancy flux is sufficiently large. In general it does play a measurable role in the flow dynamics in reducing the depth of the original layer unaffected by the intruding fluid. However, away from a relatively thin transition region between the intruding layer and the original layer, the process of penetrative entrainment does not lead to a significant reduction in the mean density (or equivalently an increase in the reduced gravity) of the intruding layer, and so a non-entraining model describes well the evolution of the bulk of the intruding layer. As the intruding layer deepens, less and less entrainment occurs. Indeed, since the total depth of the layers reduces somewhat, the plume fluid is typically dense compared to its surroundings, and so it behaves as a fountain, which eventually stalls in the intruding layer without reaching the (receding) original interface.

The penetrative entrainment can be modelled by the simple assumption that the entrainment is linearly proportional to the volume flux of the arriving plume. There is some relatively weak evidence of a higher-order dependence on Froude number (as defined in (3.3)) and direct measurement of the density of the intruding layer shows some weak non-uniformity. However, for the intermediate Froude numbers considered herein, the predictions of our simplified model are in reasonable accord with the experimental data, and therefore we believe they provide a satisfactory model of the dominant processes, with any further complexity in the penetrative entrainment model being unlikely to improve the agreement with the experimental measurements.

Fundamentally, the experimental evidence of the density profiles for intruding-flow experiments show us that there is a transition region across which the density varies smoothly between the two layers, and visual observation of the interface location using the shadowgraph technique tends to pick out the top (particularly for the interface between the intruding layer and the original layer) rather than the midpoint of the varying region. Our simple model treats the transition region as a sharp interface, and so it is important to remember that this is only an approximation to reality. To determine reasonable models to bound the actual behaviour of the flow, we have considered two situations: a simple model with penetrative entrainment where the entrainment parameter $E$ as defined in (2.16) is determined to fit best the visual observation of the interface between the two layers and a model with no penetrative entrainment at all (i.e. $E=0$ ). The evidence suggests that the fitted value of $E=1.06$ somewhat over-estimates the effect of penetrative entrainment, particularly on the density of the intruding layer, while the no-entrainment model does not capture the enhanced reduction in the depth of the original layer.

Indeed, it is best to think of the two models, one with a simple universal law for penetrative entrainment, and the other with no penetrative entrainment at all, as models which bound the region of possible flow evolution. Penetrative entrainment can be important, and our model appears to be useful to construct an upper bound on the potential impact of penetrative entrainment on flow evolution. In real applications it is likely to be difficult to distinguish between the relatively small differences in 
predictions in the model, due to other uncertainties associated with, for example, obstacles, and the fact that real sources of buoyancy are unlikely to behave exactly as point-source plumes.

The process by which a ventilating room evolves from one steady-state regime to another has some important implications for the effectiveness and response time of control systems in natural ventilation. Two situations arise which are pertinent. Consider a space heated from a localized source in which there is upflow displacement ventilation with a two-layer stratification. A control system can set the steady height of the interface between the lower layer of cold external air and the upper layer of warmer interior air by varying the ratio of the area of the upper and lower openings. In winter, the interface can be set to lie $1 \mathrm{~m}$ or so above the ground, so that the occupants lie within the comfort of the warmer upper layer (lower panels of figure 1). If the external temperature rises during the day, then the heating load may be reduced, and this will trigger the transient re-adjustment of the temperature of the upper layer of air. The continuing plume will supply a new cooler layer at the interface, causing the original hot layer to be displaced from the system. This transition will then lead to rapid re-adjustment of the temperature experienced by the occupants in the lower part of the room (middle lower panel of figure 1).

In contrast, if the external temperature falls, then this will trigger an increase in the heating load (as shown in the upper panels of figure 1). The continuing plume then rises to the top of the room, where a new warmer layer migrates downwards to the original interface. The occupants will only benefit from this new warmer layer once the original cooler layer has been eroded (right upper panel of figure 1). Our model calculations, in $\S 2$, have shown that this erosion only occurs after one or two ventilation filling box times, $T_{o}$ (as defined in (2.4)). In a real building, $T_{o}$ will be approximately 15 to 20 minutes in order that the air in the space is replenished three or four times per hour, and so with natural ventilation, an increase in the heating will only benefit the low-level occupants 15 to 20 minutes after it has been initiated. This is important in planning the operation of such a space, perhaps in constraining the time required for any pre-heating of the air.

Furthermore, the model also has relevance for air-conditioned spaces, in which cool air is supplied as downwelling plumes from high level. If one interior space is connected either to a larger space or the exterior through high- and low-level openings, then one might again expect a two-layer stratification, with a cool layer of air at the floor. If this layer becomes too cool, a reduction in the cooling load will lead to an intruding flow at intermediate level (i.e. an 'upside-down' version of the lower panels of figure 1). Occupants near ground-level in the building will only experience the warmer conditions once the original cool layer has completely drained away and the new intruding layer has propagated to the ground level. In contrast, if the space is too warm, an increase in the air-conditioning will have immediate impact in cooling the air near ground-level, as is apparent in an upside-down version of the upper panels of figure 1. This asymmetry in the response of increases and decreases in the strength of the buoyancy flux has important implications for setting the timing of the control system which manages such naturally ventilated spaces. Indeed, a more pro-active control is required in the case in which there is a delay between the change in buoyancy flux and the associated benefit in comfort conditions for the occupants.

The authors would like to thank an anonymous referee, whose queries and suggestions greatly improved our understanding of the dominant physics, and the content of the manuscript. 


\section{Appendix. Penetrative entrainment model}

To develop the model which underlies (2.16), we first propose a simple condition to determine when the rising fountain penetrates the original layer and thereby actually has the potential to entrain some of the original fluid into the intruding layer, and have $q_{e}>0$. Baines et al. (1990) collated experimental data from many authors and found that the dimensionless rise height of a fountain $\zeta_{m}$ was given by

$$
\zeta_{m}=A\left(\frac{9 \alpha \sqrt{\pi}}{10}\right)^{1 / 2} \frac{\left[m_{-}\left(\zeta_{o}\right)\right]^{3 / 4}}{\left[\hat{g}_{i} q_{-}\left(\zeta_{o}\right)-f_{-}\right]^{1 / 2}},
$$

where the (negative) buoyancy flux of the fountain at the original interface is $f_{d}=f_{-}-$ $\hat{g}_{i} q_{-}\left(\zeta_{o}\right)$ and $A=2.46$ is an empirically determined constant. More recently, Bloomfield $\&$ Kerr (2000) suggested a revised value of this coefficient to be $A=2.32 \pm 0.08$. We use the value $A=2.4$. We infer that if $\zeta_{m}<\zeta_{o}-\zeta_{i}$, then the fountain 'stalls' in the intruding layer and there is no penetrative entrainment of the upper layer.

In the case that the fountain penetrates the upper layer above $\zeta_{o}(\tau)$, it is also necessary to parameterize the amount of fluid entrained from the upper layer. We follow the proposal of Lin \& Linden (2005), and assume that the entrained volume flux is linearly proportional to the volume flux of the fountain upon arrival at the layer in which it is dense, i.e. we assume

$$
q_{e f}=E q_{d},
$$

where $E$ is assumed to be a universal, empirically determined constant, and $q_{d}$ is the volume flux arriving at the lower interface of the layer in which it is dense.

If the intruding layer is extremely thin, all the fluid entrained by the fountain is new to the intruding layer, and so $q_{e}=q_{e f}$ and $q_{d}=q_{-}\left(\zeta_{i}\right)=q_{i}$. Similarly, in some circumstances, the plume may actually still be buoyant when it reaches the new intruding layer. Now, the value of the buoyancy flux $f$ at the interface with the original upper layer may be calculated from (2.12) by substituting $q_{-}\left(\zeta_{i}\right)$ for $q_{+}\left(\zeta_{o}\right)$ and $\rho_{-}\left(\zeta_{i}\right)$ for $\rho_{+}\left(\zeta_{o}\right)$. The volume flux $q_{o}=q_{p}\left(\zeta_{o}\right)$ when the plume reaches the original layer can be calculated from (2.5), with the boundary conditions $q_{c}=q_{i+}, m_{c}=m_{i+}$. In this case, the entrained flux from the upper layer has the form $q_{e}=q_{e f}=E q_{o}$.

However, in general, the fountain is dense relative to the intruding layer, and so we adopt the fountain model from the lower interface between the lower layer of external fluid and the lower boundary of the intruding layer. With this picture, the fountain therefore entrains some of the intruding layer before entering the original layer. Baines et al. (1990) found that the fountain had a constant rate of entrainment with height, and so we assume that, provided $\zeta_{m}>\zeta_{o}-\zeta_{i}$, (2.16) applies, i.e.

$$
q_{e}=E q_{i}\left(1-\frac{\left[\zeta_{o}-\zeta_{i}\right]}{\zeta_{m}}\right)
$$

with the constant $E$ being empirically determined, as described in the main text of the paper.

\section{REFERENCES}

BAINES, W. D. 1975 Entrainment by a plume or jet at a density interface. J. Fluid Mech. 68, 309-320. Baines, W. D. \& TuRner, J. S. 1969 Turbulent buoyant convection from a source in a confined region. J. Fluid Mech. 37, 51-80.

Baines, W. D., Turner, J. S. \& CAmpbell, I. M. 1990 Turbulent fountains in an open chamber. J. Fluid Mech. 212, 557-592. 
Bolster, D. \& CAUlfield, C. P. 2008 Transients in natural ventilation - A time-periodically varying source. Building Serv. Engng Res. Technol. 29, 119-135.

Bloomfield, L. J. \& KerR, R. C. 2000 A theoretical model of a turbulent fountain. J. Fluid Mech. 424, 197-216.

Caulfield, C.-C. P. \& Woods, A. W. 1995 Plumes with nonmonotonic mixing behaviour. Geophys. Astrophys. Fluid Dyn. 79, 173-199.

Caulfield, C. P. \& Woods, A. W. 2002 The mixing in a room by a localized finite-mass-flux source of buoyancy. J. Fluid Mech. 471, 33-50.

FlynN, M. R. \& CAUlfield, C. P. 2006 Natural ventilation in interconnected chambers. J. Fluid Mech. 564, 139-158.

Gladstone, C. \& Woods, A. W. 2001 On buoyancy-driven natural ventilation of a room with a heated floor. J. Fluid Mech. 441, 293-314.

Hunt, G. R. \& KaYe, N. G. 2001 Virtual origin correction of lazy turbulent plumes. J. Fluid Mech. 435, 377-396.

Hunt, G. R. \& Linden, P. F. 2001 Steady-state flows in an enclosure ventilated by buoyancy forces assisted by wind. J. Fluid Mech. 426, 355-386.

Kaye, N. B. \& Hunt, G. R. 2004 Time-dependent flows in an emptying filling box. J. Fluid Mech. 520, $135-156$.

KumagaI, M. 1984 Turbulent buoyant convection from a source in a confined two-layered region. J. Fluid Mech. 147, 105-131.

Lin, Y. J. P. \& Linden, P. F. 2005 The entrainment due to a turbulent fountain at a density interface. J. Fluid Mech. 542, 25-52.

Linden, P. F. 1973 The interaction of a vortex ring with a sharp density interface: a model for turbulent entrainment. J. Fluid Mech. 60, 467-480.

Linden, P. F. 1999 The fluid mechanics of natural ventilation. Annu. Rev. Fluid Mech. 31, 201-238.

Linden, P. F., Lane-SerfF, G. F. \& Smeed, D. A. 1990 Emptying filling boxes: the fluid mechanics of natural ventilation. J. Fluid Mech. 212, 309-335.

McDougall, T. J. 1981 Negatively buoyant jets. Tellus 33, 313-320.

Morton, B. R. 1959 Forced plumes. J. Fluid Mech. 5, 151-163.

Morton, B. R., TAYlor, G. I. \& Turner, J. S. 1956 Turbulent gravitational convection from maintained and instantaneous sources. Proc. R. Soc. Lond. A 234, 1-23.

Settles G. S. 2001 Schlieren and Shadowgraph Techniques: Visualizing Phenomena in Transparent Media. Springer.

Woods, A. W., Caulfield, C. P. \& Phillips, J. C. 2003 Blocked natural ventilation: the effect of a source mass flux. J. Fluid Mech. 495, 119-133. 